\title{
Tetracycline-grafted PLGA nanoparticles as bone-targeting drug delivery system
}

\author{
This article was published in the following Dove Press journal: \\ International Journal of Nanomedicine \\ 8 September 2015 \\ Number of times this article has been viewed
}

\section{Hua Wang' \\ Jun Liu² \\ Shan Tao ${ }^{2}$ \\ Guihong Chai ${ }^{2}$ \\ Jianwei Wang ${ }^{3}$ \\ Fu-Qiang $\mathrm{Hu}^{2}$ \\ Hong Yuan ${ }^{2}$}

'Center of Analysis and Measurement, ${ }^{2}$ College of Pharmaceutical Sciences, Zhejiang University, Hangzhou, ${ }^{3}$ The Second Affiliated Hospital of Zhejiang University School of Medicine, Hangzhou, People's Republic of China
Correspondence: Hong Yuan College of Pharmaceutical Sciences, Zhejiang University, 866 Yuhangtang Road, Hangzhou 310058, People's

Republic of China

Tel +8657188208439

Fax +86 57I 88208439

Email yuanhong70@zju.edu.cn

Jianwei Wang

The Second Affiliated Hospital of Zhejiang University School of Medicine, 88 Jiefang Road, Hangzhou 310009 , People's Republic of China

$\mathrm{Tel}+86 \quad 15958185118$

Email wangjianwei_i@sina.com
Purpose: Nanoparticles (NPs) that target bone tissue were developed using poly(lactic-co-glycolic acid) (PLGA) copolymers and tetracycline (TC)-based bone-targeting moieties. These NPs are expected to enable the transport of drugs, such as simvastatin (SIM), for the treatment of osteoporosis.

Methods: The molecular structures of TC-PLGA were validated by ${ }^{1} \mathrm{H}-\mathrm{NMR}$, and the SIMloaded NPs were prepared using the solvent emulsification method. The surface properties, cytotoxicity, cellular uptake, cell mineralization, bone targeting potential, and animal pharmacodynamics of the TC-PLGA NPs were evaluated and compared to those of PLGA NPs.

Results: It was confirmed that the average particle size of the NPs was approximately $220 \mathrm{~nm}$. In phosphate-buffered saline (PBS, pH 7.4), the SIM-loaded NPs exhibited a cumulative release of up to $80 \%$ within 72 hours. An in vitro cell evaluation indicated that the NPs had an excellent cellular uptake capacity and showed great biocompatibility with MC3T3-E1 cells, thereby reducing the cytotoxic effects of SIM. The cell mineralization assay showed that the SIM-loaded NPs induced osteogenic differentiation and mineralized nodule formation in MC3T3-E1 cells, thereby achieving the same effect as SIM. Preliminary findings from in vitro and in vivo bone affinity assays indicated that the TC-PLGA NPs may display increased bone-targeting efficiency compared to PLGA NPs lacking a TC moiety. The use of SIM-loaded TC-PLGA NPs in treating osteoporosis was tested through animal pharmacodynamics analyses performed in ovariectomized rats, and the results suggested that the SIM-loaded TC-PLGA NPs can improve the curative effects of SIM on the recovery of bone mineral density compared to either SIMloaded PLGA NPs or SIM alone.

Conclusion: Bone-targeting NPs, which were based on the conjugation of TC to PLGA copolymers, have the ability to target bone. These NPs may be developed as a delivery system for hydrophobic drugs, and they are expected to improve the curative effects of drugs, reduce the administered drug doses, and reduce side effects in other organs.

Keywords: poly(lactic-co-glycolic acid), simvastatin, tetracycline, osteoporosis, bone targeting, nanoparticles

\section{Introduction}

Recent studies have indicated that statins have the potential ability to promote new bone formation in vitro and in animals. ${ }^{1}$ These exciting results point to a new generation of treatment options for osteoporosis, because current therapies for the treatment of osteoporosis are mostly anti-osteoclast agents, including estrogen and related compounds, bisphosphonates, calcitonin, vitamin D analogs; currently, limited methods are available to induce osteogenesis. It is known that more than $95 \%$ of gastrointestinally absorbed statins will be decomposed in the liver, resulting in a much lower concentration in the bone ${ }^{2}$ this decomposition may be the reason why most clinical studies using routine dosage of statins result in inconsistent effects on the bone. ${ }^{3,4}$ Positive results 
were obtained in the laboratory in animals orally administered with extremely high dosages of statins, ${ }^{5}$ and it has also been found that exceedingly high doses of systemically applied statins will lead to significant, adverse, and systemic side effects. ${ }^{6}$ These results have motivated us to find a suitable deliver system for statins to reduce their side effects and to target statins' delivery to the bone.

As a new type of drug delivery system, nanotechnologybased delivery systems have shown a broad variety of useful properties, such as the ability to target organs or tissues, improve drugs' biocompatibility, reduce drugs' toxic effects, and to allow controlled drug release or sustained drug release. ${ }^{7-10}$ Poly(lactic-co-glycolic acid) (PLGA) biopolymer is a hydrophobic synthetic polymer with good biodegradability and biocompatibility. It produces a biocompatible byproduct that can be eliminated through normal metabolic pathways and has been widely used in clinical treatments. Specific drugs that have very low bio-availabilities or cannot survive the environments of the gastrointestinal tract in vivo can be administered using PLGA nanoparticles (NPs) as carriers; their encapsulation into the PLGA NP delivery system not only enables their protection but also improves the bio-availability of these drugs. ${ }^{11-13}$ Some drugs with high cytotoxicity at low concentrations can be delivered with PLGA NPs, and a long-term sustained drug release can be achieved by their encapsulation into PLGA NPs. ${ }^{14}$ PLGA NPs are also a potential drug delivery device for drugs used in treating osteoporosis, and a chemical modification that targets these NPs directly to bone will be more beneficial to osteoporosis patients.

Tetracycline (TC) is a broad-spectrum antibiotic produced by the Streptomyces genus of the Actinobacteria phylum that is indicated for use against many bacterial infections. However, in clinical applications, many previous studies have shown that TC can be taken up by bone through a reaction that appears to occur directly between $\mathrm{TC}$ and hydroxyapatite $\mathrm{Ca}_{10}\left(\mathrm{PO}_{4}\right)_{6}(\mathrm{OH})_{2}$, the major constituent of bone tissue. ${ }^{15}$ Thus, TC can be a potent chemical compound for bone targeting.

In the present study, TC-PLGA was synthesized by an esterification reaction between the hydroxyl of TC and the carboxyl of PLGA. The TC-grafted PLGA NPs (TC-PLGA NPs) were prepared using the solvent emulsification method. We hoped that the TC would act as a bone targeting system that would lead the NPs directly to the bone. We chose simvastatin (SIM) as the model drug to induce the enhancement of osteogenesis. We expected the SIM-loaded TC-grafted PLGA NPs (SIM/TC-PLGA NPs) to increase the amount of
SIM that reached the bone, thereby exerting a better curative effect on osteoporosis.

\section{Materials and methods Materials}

PLGA (50:50 [the ratio of lactic acid and glycolic acid]; MW, 2,000 Da; MW =15,000 Da) was purchased from Jinan Daigang Biomaterial Co, Ltd (Jinan, Shandong, People's Republic of China). 1-(3-Dimethylaminopropyl)-3ethylcarbodiimide (EDC), hydroxybenzotriazole (HOBt), TC hydrochloride, 3-(4,5-dimethylthiazol-2-yl)-2,5-diphenyltetrazolium bromide (MTT), fluorescein isothiocyanate (FITC), $\beta$-Glycerophosphate, L-ascorbic acid, and alizarin red S were obtained from Sigma-Aldrich Co (St Louis, MO, USA). The bicinchoninic acid (BCA) protein assay kit was purchased from Beyotime Institute of Biotechnology (Haimen, Jiangsu, People's Republic of China). Alphaminimum essential medium ( $\alpha \mathrm{MEM})$, fetal bovine serum (FBS) were purchased from Thermo Fisher Scientific, Waltham, MA, USA. Hydroxyapatite powder (HAp), methylene chloride, N,N-Dimethylformamide (DMF), and polyvinyl alcohol (PVA) were purchased from Chemical Reagent Co, Ltd. (Shanghai, People's Republic of China). 1,1'-dioctadecyl-3,3,3',3'-tetramethyl indotricarbocyanine Iodide (DiR) lipophilicity fluorescence dye was obtained from Molecular Probes (Eugene, OR, USA). Hydrogenated soya phosphatidyl choline (S100) was purchased from Lipoid $\mathrm{GmbH}$ (Ludwigshafen, Germany). SIM was purchased from Zhejiang Hisun Pharmaceutical Co, Ltd (Taizhou, People's Republic of China). Otcadecylamine (ODA) was purchased from Sigma-Aldrich Co.

All mice were housed under a 12-hour light/dark cycle and had access to food and water ad libitum. All experimental protocols and animal handling procedures were performed in accordance with the US National Institutes of Health (NIH) guidelines for the care and use of laboratory animals and all experiments in the current study were approved by The Committee for Animal Experiments at Zhejiang University, Hangzhou, People's Republic of China.

\section{Synthesis and characterization of TC- PLGA $_{2000}$ copolymer}

A simple esterification reaction was used to synthesize the TC-PLGA ${ }_{2000}{ }^{16,17}$ Briefly, $500 \mathrm{mg}$ of PLGA (MW, 2,000), $156 \mathrm{mg}$ of TC hydrochloride, $72 \mathrm{mg}$ of EDC, and $50 \mathrm{mg}$ of HOBt were dissolved in $20 \mathrm{~mL}$ of DMF. This solution was heated and stirred for 24 hours at $60^{\circ} \mathrm{C}$. Then, the mixture was dialyzed against deionized water using a dialysis membrane 
(MW cut-off, 14 kDa; Spectrum Laboratories, Laguna Hills, CA, USA) for 48 hours. After centrifugation at 4,000 rpm for 10 minutes and after washing three times with deionized water, the final TC-PLGA ${ }_{2000}$ products were obtained. The ${ }^{1} \mathrm{H}-\mathrm{NMR}$ spectra of the products were obtained using a nuclear magnetic resonance spectrometer (BioSpin AC-80; Bruker Optik GmbH, Ettlingen, Germany). TC-PLGA ${ }_{2000}$ was dissolved in deuterated dimethyl sulfoxide (DMSO) at $50 \mathrm{mg} / \mathrm{mL}$. PLGA ${ }_{2000}$ and TC were compared at a concentration of $20 \mathrm{mg} / \mathrm{mL}$.

\section{Preparation of fluorescein}

A reaction between the amino group of ODA and the isothiocyanate group of FITC was used to synthesize the ODA-FITC. Briefly, $20 \mathrm{mg}$ of ODA and $28 \mathrm{mg}$ of FITC were dissolved in 6 $\mathrm{mL}$ of ethanol. The solution was heated and stirred for 24 hours at $50^{\circ} \mathrm{C}$, protected from light. After the reaction was finished, the mixture was mixed with $50 \mathrm{~mL}$ of distilled water to precipitate the ODA-FITC. The precipitate was filtrated through a $0.45 \mathrm{~mm}$ filter (EMD Millipore, Billerica, MA, USA) and was washed three times with distilled water. The final product was lyophilized and stored in the dark for further use.

\section{Preparation of PLGA NPs and TC-PLGA NPs}

Over all, $5 \mathrm{mg}$ of SIM and $10 \mathrm{mg}$ of hydrogenated soya phosphatidyl choline (S100) were dissolved in $1.0 \mathrm{~mL}$ of methylene chloride and conjugated by stirring for 30 minutes. The solution was then evaporated under vacuum at room temperature. After all of the methylene chloride was evaporated, the final SIM-S100 drug compound was obtained.

PLGA NPs and TC-PLGA NPs were prepared via the solvent emulsification method. ${ }^{18,19}$ Briefly, the SIM-S100 drug compound (15 mg; only for SIM-loaded PLGA NPs and TC-PLGA NPs); ODA-FITC (5 mg; only for the ODA-FITC-labeled PLGA NPs and the TC-PLGA NPs); DiR (1.0 mg; only for the DiR-labeled PLGA NPs and the TC-PLGA NPs), $80 \mathrm{mg}$ PLGA (MW =15,000); and $20 \mathrm{mg}$ TC-PLGA ${ }_{2000}$ (only for the TC-PLGA NPs), or the PLGA ${ }_{2000}$ (only for the PLGA NPs) were dissolved in $2 \mathrm{~mL}$ methylene chloride. The mixture was quickly dispersed into $10 \mathrm{~mL}$ of PVA solution $(2 \% ; \mathrm{w} / \mathrm{v})$ under high shear and dispersing at $20,000 \mathrm{rpm}$ for 10 minutes, followed by homogenization via a probe sonicator. The emulsion was then evaporated under vacuum until almost all of the methylene chloride was evaporated. The PLGA NPs and TC-PLGA NPs were isolated by centrifugation at 20,000 rpm for 20 minutes and were washed with deionized water several times. The final PLGA NPs or TC-PLGA NPs were redispersed in deionized water.

\section{High-performance liquid chromatography assay for SIM}

High-performance liquid chromatography (HPLC) analysis of SIM was performed using a modular liquid chromatograph system (Agilent Technologies, Santa Clara, CA, USA) with a C18 analytical column (Dikma Technologies, Lake Forest, CA, USA ) column $(250 \mathrm{~mm} \times 4.6 \mathrm{~mm} ; 5 \mu \mathrm{m})$. The mobile phase was a mixture of acetonitrile and water containing $25 \mathrm{mM}$ phosphoric acid $(65: 35 ; \mathrm{v}: \mathrm{v})$, eluted at a flow rate of $1.0 \mathrm{~mL} / \mathrm{min}$ and a sample injection volume of $100 \mu \mathrm{L}$. The UV detector wave length was set at $239 \mathrm{~nm} .^{20}$

\section{Characterization of PLGA NPs and TC-PLGA NPs \\ Determination of particle size}

The particle sizes of the PLGA NP and TC-PLGA NP suspensions $(1.0 \mathrm{mg} / \mathrm{mL})$ were determined by laser scattering technique using a Zetasizer analyzer $(3000 \mathrm{HS}$; Malvern Instruments Ltd).

\section{Transmission electron microscopy observations}

The morphological characteristics of the PLGA NPs and TC-PLGA NPs were examined using transmission electron microscopy (TEM; JEM-1230; JEOL, Tokyo, Japan). The samples were put on copper grids and were stained with $2 \%$ (w/v) phosphotungstic acid.

\section{Determination of drug entrapment efficiency}

The SIM-loaded PLGA NPs and SIM-loaded TC-PLGA NPs were destroyed by DMSO. The SIM content was measured by HPLC, and the SIM content in DMSO $\left(\mathrm{C}_{\mathrm{s}} ; \mathrm{mg} / \mathrm{mL}\right)$ was determined. The drug loading (DL\%) of SIM and the entrapment efficiency (EE\%) were calculated using Equations 1 and 2 , respectively:

$$
\mathrm{DL} \%=\frac{\mathrm{C}_{\mathrm{s}} \times \mathrm{V}}{\mathrm{C}_{\mathrm{s}} \times \mathrm{V}+\mathrm{W}_{0}} \times 100 \%
$$

and

$$
\mathrm{EE} \%=\frac{\mathrm{C}_{\mathrm{s}} \times \mathrm{V}}{\mathrm{W}_{\mathrm{s}}} \times 100 \%
$$

where $\mathrm{W}_{\mathrm{s}}$ represents the weight of SIM $(\mathrm{mg}), \mathrm{W}_{0}$ represents the weight of the synthetic polymer (mg) added into the system, and $\mathrm{V}$ denotes the volume of the DMSO (mL). 


\section{Drug release kinetics in vitro}

The dialysis bag diffusion technique was used to study in vitro drug release from PLGA NPs and TC-PLGA NPs. ${ }^{21}$ The SIM-loaded PLGA NPs or TC-PLGA NP suspension equivalent to $50 \mu \mathrm{g}$ SIM were added until they filled the dialysis bag (MW cut-off, $14 \mathrm{KDa}$; Spectrum Laboratories, Laguna Hills, CA, USA) and were placed in $15 \mathrm{~mL}$ of PBS ( $\mathrm{pH}$ 7.4), were stirred at $60 \mathrm{r} / \mathrm{min}$, and were maintained at a temperature of $37^{\circ} \mathrm{C}$. At predetermined time intervals (ie, $1,2,4,6,9,12,24,36,48$, and 72 hours), the dissolution medium was completely removed for analysis and was replaced with an equal volume of fresh dissolution medium. HPLC was used to measure the drug concentration.

\section{Cell culture and evaluation}

\section{MC3T3-EI cell culture}

MC3T3-E1 cells, a clonal pre-osteoblastic cell line derived from newborn mouse calvaria, which were obtained from Keduo Biochemistry Co, Ltd (Hangzhou, People's Republic of China), were cultured in $\alpha$ MEM containing $10 \%(\mathrm{v} / \mathrm{v})$ FBS. When the cells were seeded for the osteogenic differentiation experiment, the basic medium was supplemented with $10 \mathrm{mmol} / \mathrm{L} \beta$-glycerophosphate and $50 \mu \mathrm{g} / \mathrm{L} \mathrm{L}$-ascorbic acid. The medium was changed every 2 days. ${ }^{22}$

\section{Cytotoxicity of PLGA NPs and TC-PLGA NPs}

To determine the concentration of SIM that did not induce cytotoxicity, $200 \mu \mathrm{L}$ of MC3T3-E1 cells were plated at a density of $2 \times 10^{4}$ cells $/ \mathrm{mL}$ in a 96-well plate and were cultured with SIM-loaded PLGA NPs and TC-PLGA NPs for 48 hours. The concentrations of SIM loaded into the NPs ranged from $10^{-8} \mathrm{~mol} / \mathrm{L}$ to $10^{-5} \mathrm{~mol} / \mathrm{L}$. After 48 hours of treatment, cell proliferation was quantified using the MTT assay. Briefly, $20 \mu \mathrm{L}$ of MTT solution $(5 \mathrm{mg} / \mathrm{mL})$ was added, followed by incubation for another 4 hours. After MTT-containing medium was removed and formazan was solubilized in $200 \mu \mathrm{L}$ DMSO, The optical density values of solution were measured at a wavelength of $570 \mathrm{~nm}$, and cell viability was calculated by the following equation: ${ }^{23}$

$$
\text { Cell viability }(\%)=\frac{\text { OD570 }(\text { sample })}{\text { OD570 (control) }} \times 100 \% \text {. }
$$

\section{Cellular uptake investigation}

For the cellular uptake study, MC3T3-E1 cells were plated in a 12-well plate at a density of $5 \times 10^{4}$ cells/well and were then treated with fresh $\alpha$ MEM containing ODA-FITC-loaded
PLGA NPs and TC-PLGA NPs $(100 \mu \mathrm{g} / \mathrm{mL})$ for 24 hours at $37^{\circ} \mathrm{C}$. At pre-determined intervals (ie, $2,4,7,10$ and 24 hours), the cells were washed three times with PBS and were collected. Then, the collected cells were subjected to repeated freezing and thawing with DMSO. After they were centrifuged at $10,000 \mathrm{rpm}$ for 5 minutes, the fluorescence of supernatants was detected by fluorescence spectrophotometer and a Micro BCA protein assay kit was used to determine the intracellular protein contents. The cellular uptake percentage of the fluorescent loaded NPs was calculated by the following equation:

$$
\begin{aligned}
& \text { NPs uptake } \\
& \text { percentage (\%) }
\end{aligned}=\frac{\mathrm{C}_{\mathrm{t}}}{\mathrm{C}_{\mathrm{t} 0}} \times 100 \%
$$

where $\mathrm{C}_{\mathrm{t}}$ presents the intracellular ODA-FITC concentration at times $t$, and $\mathrm{C}_{\mathrm{t} 0}$ denotes the initial ODA-FITC concentration at times $\mathrm{t}_{0}$.

\section{Alizarin red S assay}

To confirm the mineralization in differentiated cells, MC3T3-E1 cells were plated in a 12-well plate at a density of $5 \times 10^{4}$ cells/well and were treated with osteogenic differentiation medium containing SIM-loaded PLGA NPs and TC-PLGA NPs $\left(10^{-8} \mathrm{~mol} / \mathrm{mL}\right.$ or $10^{-7} \mathrm{~mol} / \mathrm{mL}$ of SIM equivalent) for 14 days. The osteogenic differentiation medium was refreshed every other day. After 14 days, $1.5 \%$ alizarin red $\mathrm{S}$ was prepared in distilled water, and the $\mathrm{pH}$ was adjusted to $4.0-4.2$ using $0.5 \%$ ammonium hydroxide. Differentiated MC3T3-E1 cells were fixed with 70\% ethanol (1 hour), were washed, and were stained with alizarin red S for 10-15 minutes. After the removal of the unreacted dye, the mineralized nodules were identified as red spots. Then, the alizarin red $\mathrm{S}$ staining was released from the cell matrix by incubating the samples in $10 \%$ cetylpyridinium chloride for 15 minutes. The alizarin red $\mathrm{S}$ concentration was determined by measuring the absorbance at $562 \mathrm{~nm} .^{24,25}$

\section{Bone mineral binding ability in vitro}

The adhesion between the bone-like substrates and the bonetargeting NPs was demonstrated in vitro. The binding capacity of NPs to HAp was evaluated using a method similar to that described in some previous reports. ${ }^{26,27}$ In brief, NPs loaded with the hydrophobic fluorescent dye ODA-FITC were used at a dilution of $4 \mathrm{~mL}(0.8 \mathrm{mg} / \mathrm{mL}$ of NPs equivalent $)$. Their initial fluorescence emission intensities were measured (Ex [excitation wavelength] $=516 \mathrm{~nm}$, Em [emission wavelength $]=532 \mathrm{~nm}$, slit [width of slit] $=5 \mathrm{~nm}, \mathrm{U}$ [voltage of the photomultiplier] $=700 \mathrm{~V}$ ) using a fluorescence spectrometer 
(F-2500; Hitachi Ltd, Tokyo, Japan). Then, 25 mg of HAp with $\mathrm{Ca}_{10}\left(\mathrm{PO}_{4}\right)_{6}(\mathrm{OH})_{2}$ was added to $4 \mathrm{~mL}$ each of the ODA-FITCloaded NPs. These suspensions were shaken under mechanical stirring at $60 \mathrm{rpm}$ for 3 hours to allow for the adsorption of the ODA-FITC-loaded NPs onto the HAp. The amount of the ODA-FITC-labeled NPs adsorbed was determined by measuring the difference between the fluorescence emission intensity of the initial stock solution and that of the supernatant after adsorption, according to the following equation:

$$
\begin{aligned}
& \text { HAp absorption } \\
& \text { affinity }
\end{aligned}=\frac{I_{t 0}-I_{t}}{I_{t 0}} \times 100 \%
$$

where $I_{t 0}$ and $I_{t}$ are the fluorescence emission intensities of the initial NP stock solution and that of the supernatant after 3 hours adsorption, respectively.

\section{In vivo evaluation of the bone-targeting NPs in mice}

The in vivo bone-targeting capacities of PLGA NPs and TC-PLGA NPs labeled with DiR, a lipophilic fluorescence dye, were evaluated in US Institute of Cancer Research (ICR) mice. The ICR mice were divided into two groups, one of which was administered with the PLGA NPs (the control) and the other with the bone-targeting TC-PLGA NPs. For each experiment, three animals per group were used, and $0.2 \mathrm{~mL}$ of the NPs ( $4 \mathrm{mg} / \mathrm{mL}$ ) was injected into the tail veins of each mouse. After the injection, all the animals had free access to food and water. After 48 hours, the animals were sacrificed, and the major organs (heart, liver, spleen, lung, kidney, femur, tibia, and fibula) were collected. The fluorescent signals in these organs from three mice in each group were detected using the Maestro EX in vivo imaging system (Cambridge Research \& Instrumentation, Inc, Woburn, MA, USA). The distribution of the fluorescent intensities as a percentage of the NPs was calculated from the following equation:

$$
\begin{aligned}
& \text { Distribution of fluorescent } \\
& \text { intensities (\%) }
\end{aligned}=\frac{\mathrm{A}_{\text {tissue }}}{\mathrm{A}_{5 \%} \times 20} \times 100 \%
$$

where $\mathrm{A}_{\text {tissue }}$ represents the fluorescent intensities of the major tissues and $\mathrm{A}_{5 \%}$ represents the fluorescent intensities of DiR-loaded NPs in suspension at the same injected dose at a concentration of $5 \%$.

\section{Animal pharmacodynamics to evaluate NP bone targeting}

Twenty female Sprague Dawley rats (200-250 g each) were used. The animals were randomly assigned to five groups of four animals per treatment group as follows: group 1 control sham-operated rats (SHAM); group 2, ovariectomized rats (OVX); group 3, ovariectomized rats that were intravenously injected with SIM at a dose of $0.5 \mathrm{mg} / \mathrm{kg} / 2$ days (OVX + SIM); group 4, ovariectomized rats that were intravenously injected with SIM-loaded PLGA NPs at a dose of $0.5 \mathrm{mg} / \mathrm{kg} / 2$ days (OVX + SIM-loaded NPs); and group 5, ovariectomized rats that were intravenously injected with SIM-loaded TCPLGA NPs at a dose of $0.5 \mathrm{mg} / \mathrm{kg} / 2$ days (OVX + SIM-loaded TC-NPs). All rats except those in group 1 were bilaterally ovariectomized under anesthesia using pentobarbital sodium $(40 \mathrm{mg} / \mathrm{kg}),{ }^{28}$ whereas the rats of group 1 were subjected to a sham operation under the same conditions of anesthesia and abdominal invasion. The injection of SIM-loaded NPs started 1 month after the bilateral ovariectomy or sham operation and continued for 2 months. After 2 months of injection, all animals were sacrificed. The left femurs of the animals were isolated and the bone mineral density (BMD) was measured using a dual-energy X-ray absorptiometer (GE Healthcare Bio-Sciences Corp., Piscataway, NJ, USA). ${ }^{29,30}$ The repair of BMD as a percentage of the total bone was calculated from the following equation:

$$
\begin{aligned}
& \text { Recovery of bone } \\
& \text { mineral density (\%) }
\end{aligned}=\frac{\mathrm{BMD}_{\text {drug }}-\mathrm{BMD}_{\mathrm{ovx}}}{\mathrm{BMD}_{\text {sham }}-\mathrm{BMD}_{\mathrm{ovx}}}
$$

where $\mathrm{BMD}_{\text {drug }}, \mathrm{BMD}_{\mathrm{ovx}}$ and $\mathrm{BMD}_{\text {sham }}$ represent the $\mathrm{BMD}$ of bone belonging to the rats of the administration, OVX, and SHAM groups, respectively.

\section{Statistical analysis}

The data were expressed as the mean \pm standard deviation (SD) unless noted otherwise. The statistical analyses of the data were performed using Student's $t$-test. Differences characterized by $P<0.05$ were considered statistically significant.

\section{Results and discussion}

\section{Synthesis and characterization of TC-PLGA ${ }_{2000}$ copolymers}

The TC-PLGA 2000 was successfully synthesized by the direct conjugation of the carboxyl group of PLGA $_{2000}$ with the hydroxyl group of TC via an esterification reaction, as described in Figure 1. To characterize the TC-PLGA ${ }_{2000}$, the product was evaluated by ${ }^{1} \mathrm{H}-\mathrm{NMR}$ spectroscopy. As shown in Figure 2A, the ${ }^{1} \mathrm{H}-\mathrm{NMR}$ spectra revealed peaks at $7.0-8.0 \mathrm{ppm}$ that corresponded to the hydrogens of the benzene ring of TC. In Figure 2B, the peak at 4.0-6.0 ppm 


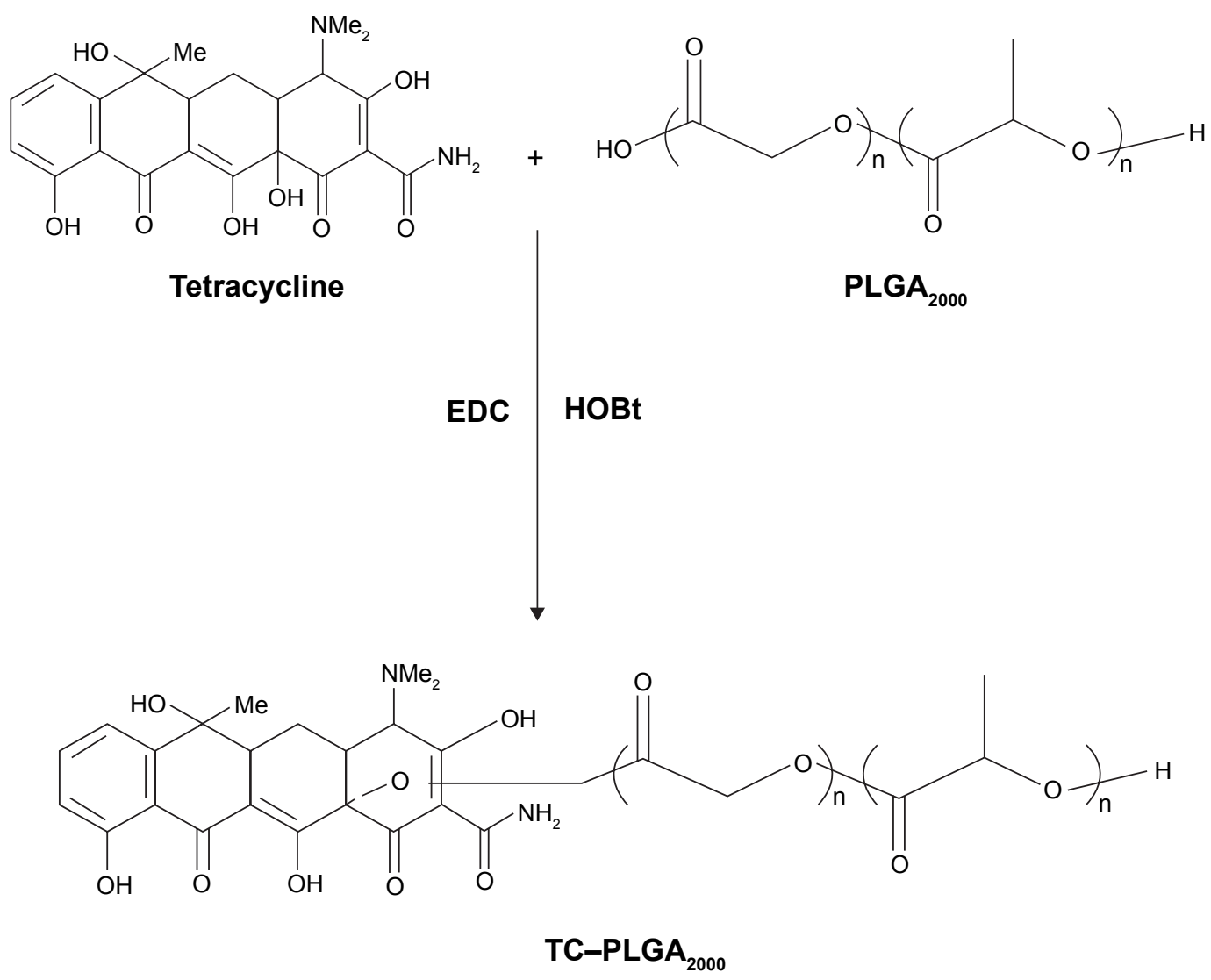

Figure I Synthesis route of TC-PLGA ${ }_{2000}$.

Abbreviations: PLGA, poly(lactic-co-glycolic acid); TC-PLGA ${ }_{2000}$, tetracycline-poly(lactic-co-glycolic acid) moiety; EDC, I-(3-dimethylaminopropyl)-3-ethylcarbodiimide; $\mathrm{HOBt}$, hydroxybenzotriazole.

represents the methylene and methylidyne hydrogens of the PLGA copolymers. In Figure 2C, we observed all of the above-mentioned characteristics, as well as a peak at $13 \mathrm{ppm}$ corresponding to the carboxyl of PLGA, which indicated that the carboxyl group of PLGA $_{2000}$ had conjugated with the hydroxyl group of TC. These results all indicated that the synthesis of the TC-PLGA 2000 was successful.

\section{Properties of blank and SIM-loaded PLGA NPs and TC-PLGA NPs}

As shown in Table 1, the sizes of the blank PLGA NPs, SIMloaded PLGA NPs and TC-PLGA NPs were approximately $220 \mathrm{~nm}$. The TC graft had no significant effect on the particle size $(P>0.05)$. The polydispersity index (PI) of these particles was approximately 0.3 . These results demonstrated the narrow size distribution of the NPs.

The NP size was further confirmed by using TEM. As shown in Figure 3, the blank and SIM-loaded NPs were all spherical or spherical-like particles, and were uniform in size and shape, as shown in TEM image (Figure 3). There were no obvious changes in particle size after TC graft.
The DL $\%$ and EE\% are shown in Table 1 . The EE\% of the SIM-loaded PLGA NPs was $>75 \%$. There were no obvious changes in EE\% after TC graft $(P>0.05)$.

The in vitro drug release behaviors of SIM-loaded NPs are shown in Figure 4. The SIM-loaded PLGA NPs exhibited a cumulative drug release $>80 \%$ during a 72 -hour time span. There were no significant differences in drug release profiles between the normal PLGA NP group and the TC-PLGA NP group $(P>0.05)$. Hence, the TC graft could be considered to have no effect on drug release.

\section{Cell culture and evaluation Cytotoxicity of NPs}

Figure 5A shows the results of an MTT assay used to assess the cytotoxic effects of the PLGA NPs and TC-PLGA NPs on MC3T3-E1 cells. The results in Figure 5A demonstrate that the PLGA NPs and TC-PLGA NPs had no cytotoxicity, and that cell viability was nearly $100 \%$ at PLGA and TC-PLGA NP concentrations $<100 \mu \mathrm{g} / \mathrm{mL}$.

To determine the optimum concentration of SIMloaded NPs for osteogenic differentiation, we cultured 

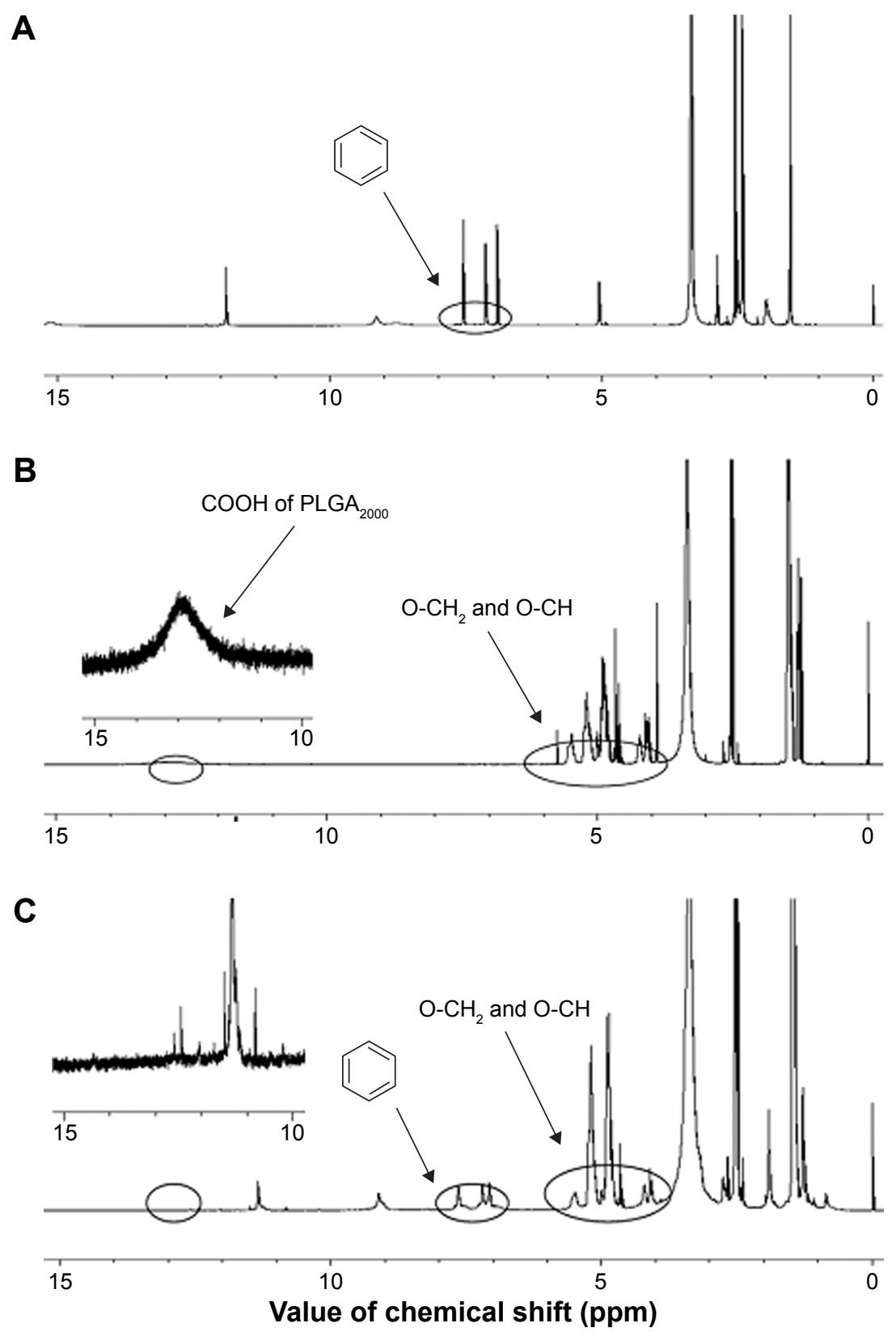

Figure 2 'H-NMR spectra of TC (A), PLGA 2000 (B), and TC-PLGA 2000 (C) samples.

Note: The peaks in the ovals signify the structures of methylene and methylidyne hydrogens of the PLGA copolymers, and hydrogens of the benzene ring of TC, respectively.

Abbreviations: 'H-NMR, hydrogen spectra of nuclear magnetic resonance; TC, tetracycline; PLGA, poly(lactic-co-glycolic acid); TC-PLGA ${ }_{2000}$, tetracycline-poly(lactic-coglycolic acid) moiety; ppm, part per million.

the MC3T3-E1 cells with varying concentrations of SIMloaded NPs. As seen in Figure 5B, SIM-loaded NPs could reduce the cytotoxic effects and improve the biocompatibility of SIM, especially when the concentration of SIM was $>5 \times 10^{-6} \mathrm{~mol} / \mathrm{L}$. Meanwhile, the cytotoxic effects of the SIM-loaded PLGA NPs and the SIM-loaded TC-PLGA NPs were not significantly different, indicating that the TC graft had a negligible effect on cell viability.

When the concentrations of SIM were $>5 \times 10^{-7} \mathrm{~mol} / \mathrm{L}$, the cells treated with either SIM or SIM-loaded NPs displayed significant cytotoxic effects, resulting in a decrease in cell viability compared to the untreated control cells $(P<0.05)$. These results suggested that the concentration of SIM should be kept below $10^{-7} \mathrm{~mol} / \mathrm{L}$ in future experiments testing osteogenic differentiation.

\section{Cell uptake investigation}

Cellular uptake behaviors of PLGA NPs and TC-PLGA NPs were evaluated using MC3T3-E1 cells as model cells. ODA-FITC, which was water-insoluble and had almost no 
Table I Properties of blank and SIM-loaded PLGA NPs and TC-PLGA NPs

\begin{tabular}{lllll}
\hline Carriers & Size $(\mathbf{n m})$ & PI & EE\% & DL\% \\
\hline PLGA NPs & $215.3 \pm 12.4$ & $0.29 \pm 0.01$ & - & - \\
TC-PLGA NPs & $216.5 \pm 4.9$ & $0.31 \pm 0.01$ & - & - \\
SIM/PLGA NPs & $225.4 \pm 11.3$ & $0.33 \pm 0.02$ & $80.97 \pm 3.96$ & $3.89 \pm 0.18$ \\
SIM/TC-PLGA NPs & $224.9 \pm 10.3$ & $0.28 \pm 0.01$ & $75.92 \pm 2.12$ & $3.65 \pm 0.10$ \\
\hline
\end{tabular}

Note: Data represent the mean \pm standard deviation $(n=3)$.

Abbreviations: PI, polydispersity index of the particle size; EE\%, entrapment efficiency in percentages; DL\%, drug loading in percentages; NPs, nanoparticles; TC, tetracycline; PLGA, poly(lactic-co-glycolic acid); TC-PLGA, tetracycline-poly(lactic-co-glycolic acid) moiety; SIM, simvastatin.

release in culture medium, was used as a label to quantify the internalization of the NPs into the cells. As shown in Figure 6, the uptake of NPs by MC3T3-E1 cells increased with passing time. After 24 hours, the uptake percentage was approximately $8.91 \%$ for the ODA-FITC-loaded PLGA NPs but was just $6.67 \%$ for the ODA-FITC-loaded TC-PLGA NPs, which also showed a weak decline over time. This may have been because the TC graft on the surface of the PLGA NPs may have improved the hydrophilicity of the
PLGA NPs, leading to a decrease in the ability of the NPs to be internalized.

\section{Effects of SIM-loaded NPs on mineralized nodule formation}

We tested the effects of SIM-loaded NPs on osteoblast differentiation, as evidenced by mineralization. MC3T3-E1 cells were cultured in osteogenic differentiation medium for 14 days with SIM-loaded PLGA NPs and SIM-loaded
A

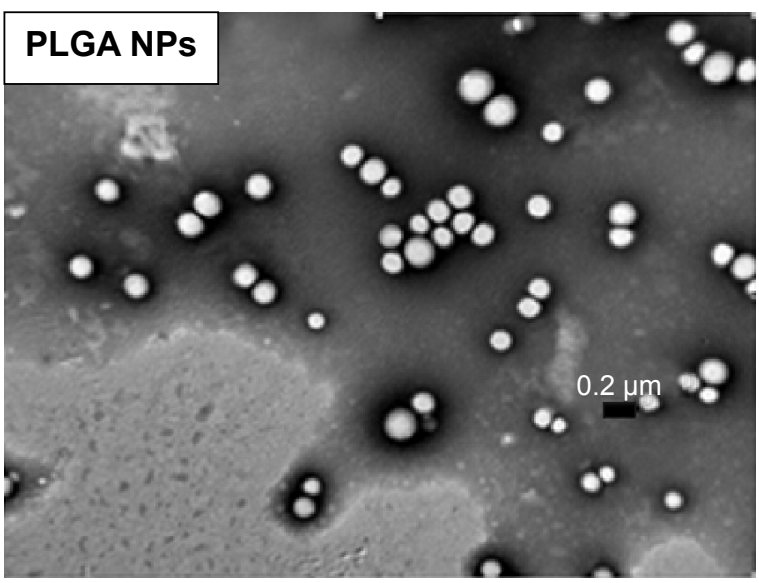

C

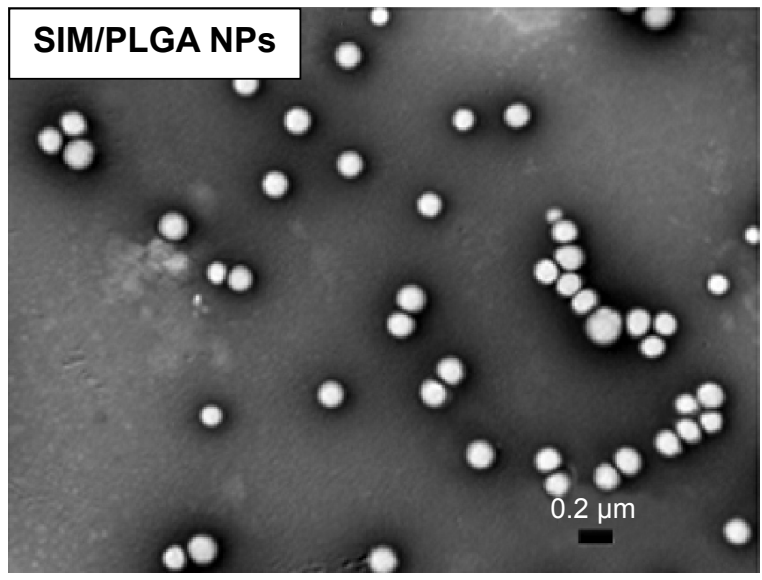

B

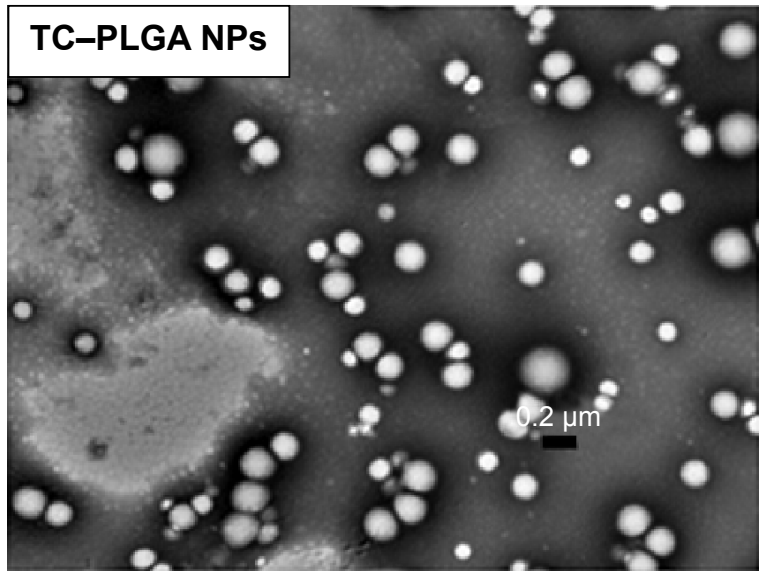

D

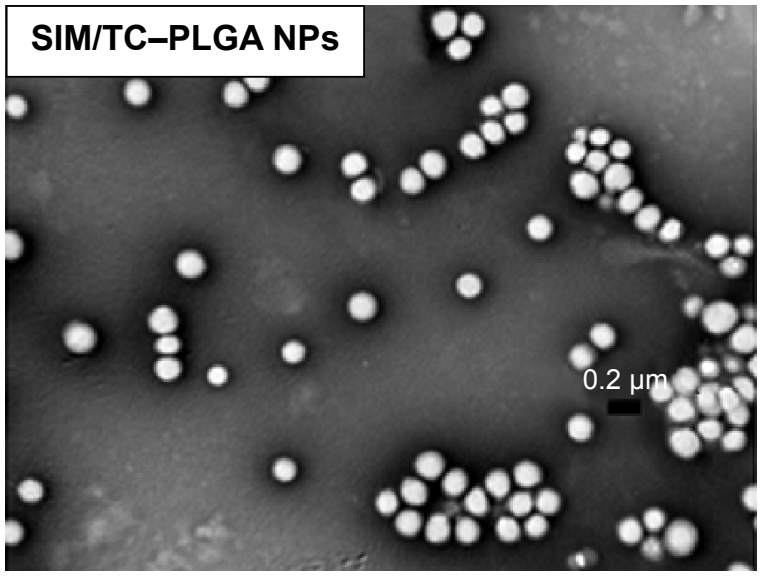

Figure 3 TEM images of blank NPs and SIM-loaded PLGA or TC-PLGA NPs: PLGA NPs (A); TC-PLGA NPs (B); SIM-loaded PLGA NPs (C); and SIM-loaded TC-PLGA NPs (D).

Abbreviations: TEM, transmission electron microscopy; NPs, nanoparticles; SIM, simvastatin; PLGA, poly(lactic-co-glycolic acid); TC-PLGA, tetracycline-poly(lactic-coglycolic acid) moiety. 


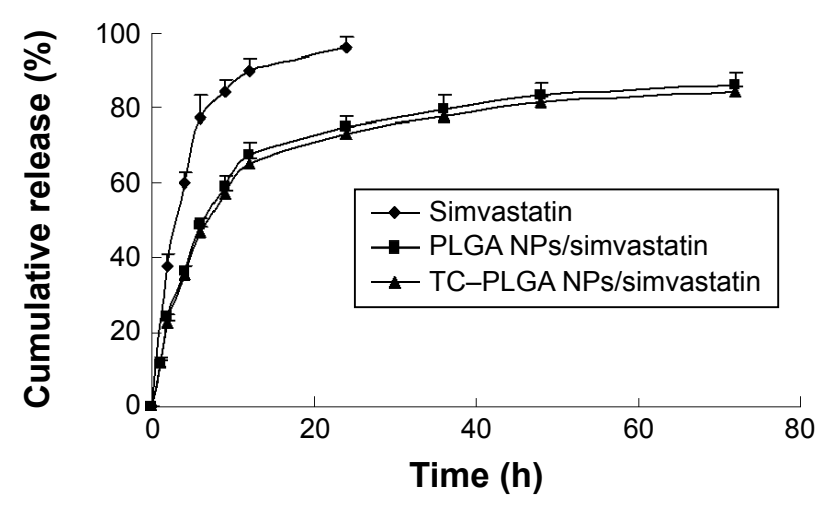

Figure 4 In vitro release profiles of SIM from different formulations.

Note: The data are plotted as the mean \pm SD $(n=3)$.

Abbreviations: NPs, nanoparticles; PLGA, poly(lactic-co-glycolic acid); SIM, simvastatin; TC-PLGA, tetracycline-poly(lactic-co-glycolic acid) moiety; h, hours; SD, standard deviation.
TC-PLGA NPs. The medium used in our in vitro experiments to induce osteogenic differentiation contained $50 \mu \mathrm{g} / \mathrm{mL}$ ascorbic acid and $10 \mathrm{mM} \beta$-glycerophosphate. This is a widely employed standard medium in osteogenic differentiation studies ${ }^{24,25}$ and has been reported to possess osteogenic activities. ${ }^{31}$ Due to its necessary involvement in the cell culture studies, we also examined the osteogenic effects of this medium, and we used differentiation medium treatment as a background in order to assess the actual osteogenic activities of the SIM-loaded NPs.

As shown in Figure 7, treatments with free SIM and SIM-loaded NPs all yielded an increase in cell mineralization over that of the differentiation medium-treated cells. The increase in the activity was significant both at SIM

A
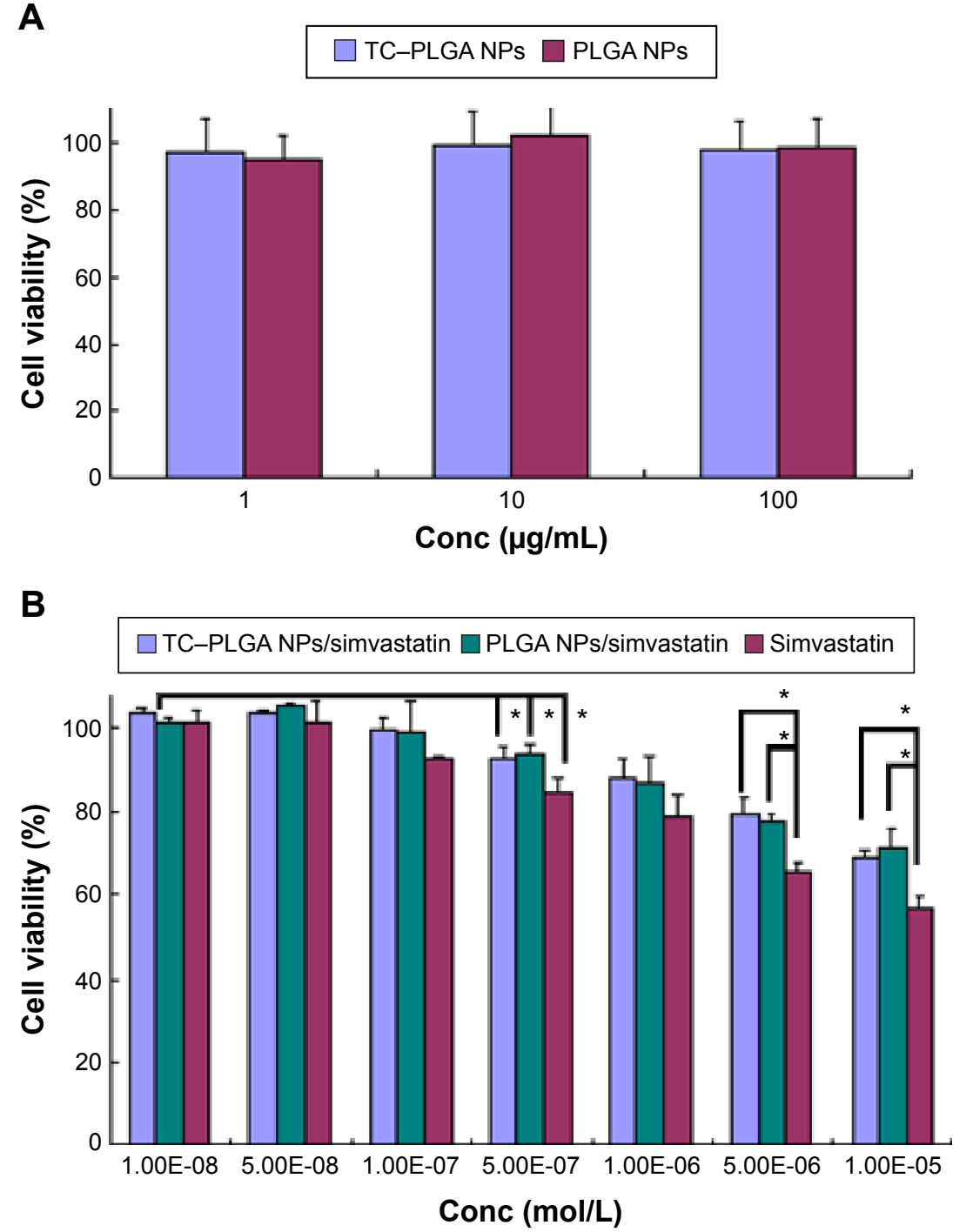

Figure 5 Cytotoxic effects of PLGA NPs and TC-PLGA NPs against MC3T3-EI cells at 48 hours (A). Cytotoxic effects of SIM, SIM-loaded PLGA NPs, and SIM-loaded TC-PLGA NPs against MC3T3-EI cells at 48 hours (B).

Notes: The data are plotted as the mean \pm SD $(n=3)$. Significant differences against the control, $* P<0.05$.

Abbreviations: NPs, nanoparticles; PLGA, poly(lactic-co-glycolic acid); SIM, simvastatin; TC-PLGA, tetracycline-poly(lactic-co-glycolic acid) moiety; Conc, concentration; $\mathrm{SD}$, standard deviation. 
A
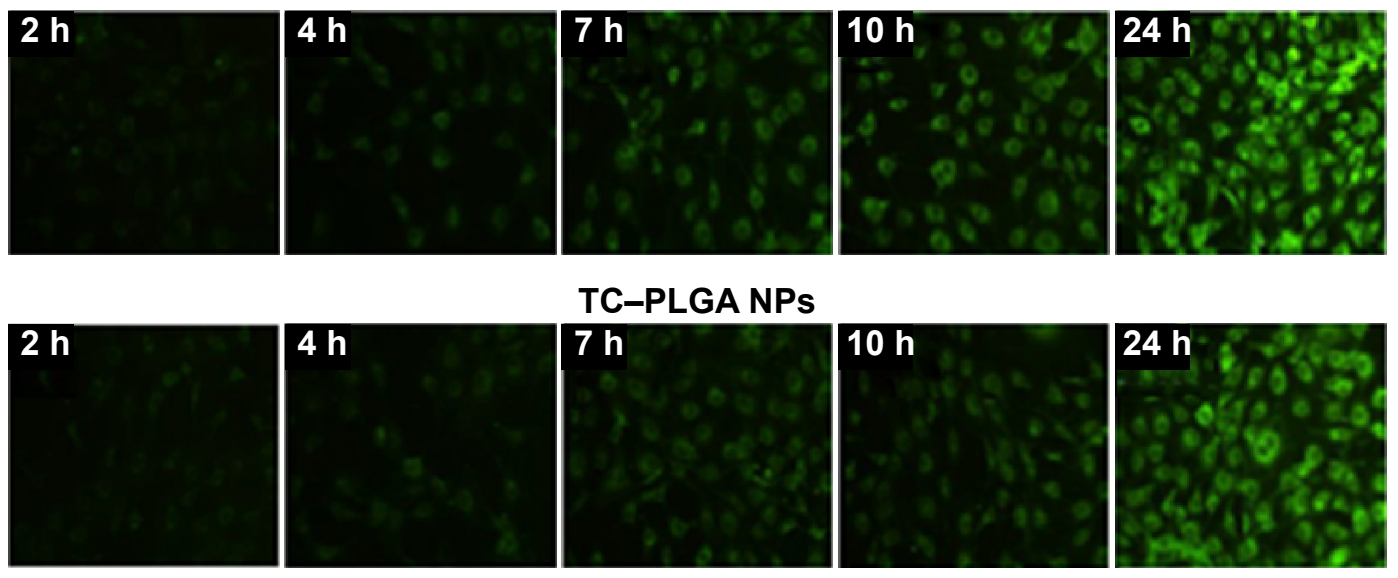

TC-PLGA NPs
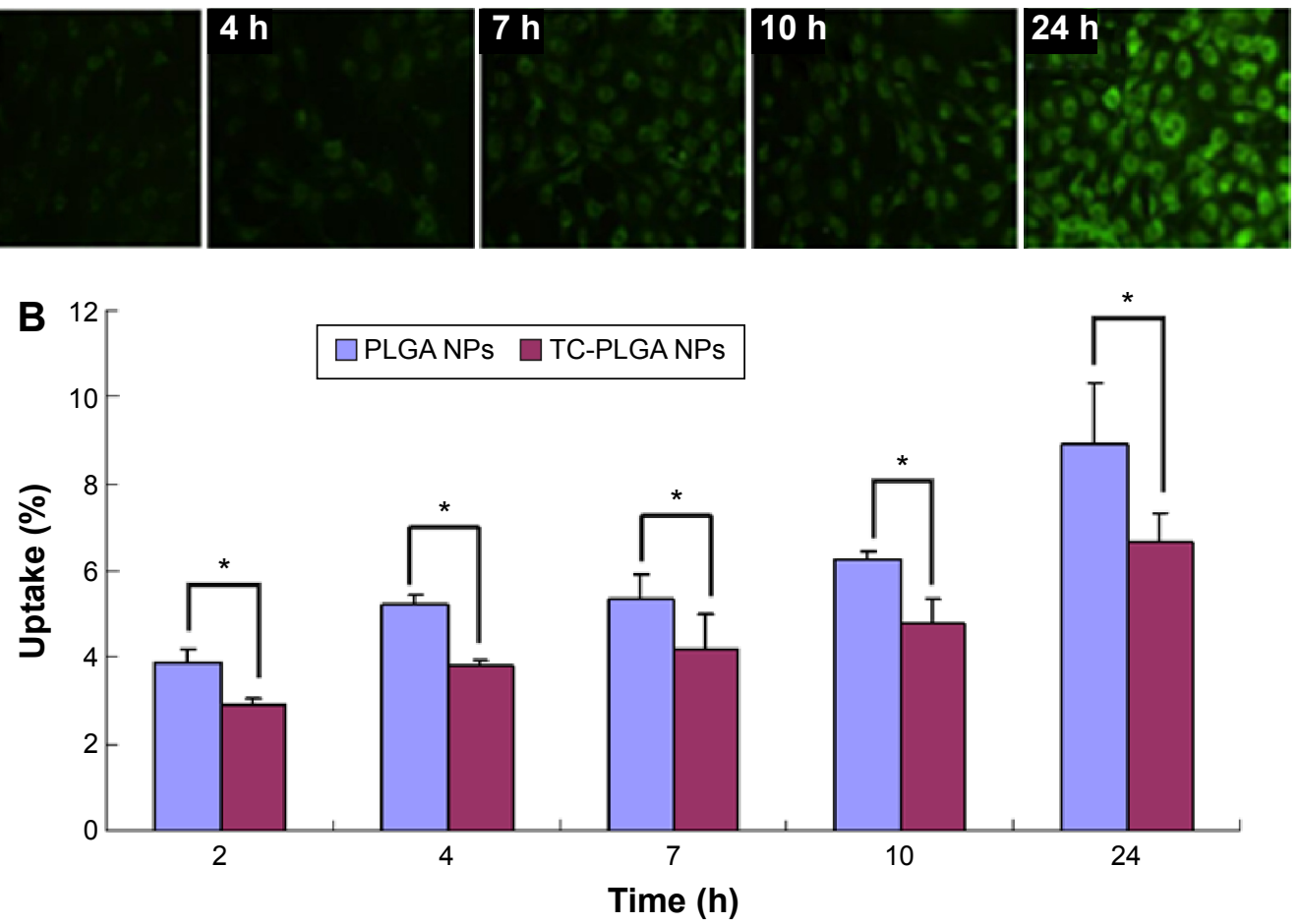

Figure 6 (A) Fluorescence images of NPs' uptake in MC3T3-EI cells after cells were incubated with ODA-FITC/PLGA NPs and ODA-FITC/TC-PLGA NPs for different times. (B) Quantitative uptake percentage of ODA-FITC/PLGA NPs and ODA-FITC/TC-PLGA NPs in MC3T3-EI cells for different times.

Notes: Data represent the mean \pm SD $(n=3)$. Significant differences against the control, $* P<0.05$. (A) The magnification is $200 \times$.

Abbreviations: NPs, nanoparticles; PLGA, poly(lactic-co-glycolic acid); ODA, otcadecylamine; TC-PLGA, tetracycline-poly(lactic-co-glycolic acid) moiety; FITC, fluorescein isothiocyanate; SD, standard deviation; h, hours.

concentrations of $10^{-8} \mathrm{~mol} / \mathrm{L}$ and $10^{-7} \mathrm{~mol} / \mathrm{L}$, though the SIM concentration of $10^{-7} \mathrm{~mol} / \mathrm{L}$ had a better effect on cell mineralization. The effects of SIM, SIM-loaded PLGA NPs, and SIM-loaded TC-PLGA NPs on the formation of mineralized nodules were not significantly different at the same concentrations, indicating that the interference of the PLGA NPs and TC-PLGA NPs with osteogenic differentiation was negligible.

\section{Bone mineral binding ability}

Apatite is the main component of bone in its natural state. In vertebrate bones, this inorganic material is known as hydroxyapatite $\mathrm{Ca}_{10}\left(\mathrm{PO}_{4}\right)_{6}(\mathrm{OH})_{2}$, or carbonated hydroxyapatite. A HAp binding assay was performed in vitro (Figure 8A), and the affinity of the bone-targeting NPs for HAp are shown in Figure 8B. The data presented in Figure 8B clearly demonstrate that the proportion of the TC-PLGA NPs bound to HAp (87.94\%) was greater than the proportion of bound PLGA NPs (18.59\%). These results indicated that after grafting, TC could still maintain its affinity for HAp and that the ability for bone targeting of PLGA NPs was improved.

\section{In vivo distribution assay}

An in vivo biodistribution assay in ICR mice was performed to further examine the bone-targeting ability of TC-PLGA NPs. Figure 9B shows the accumulation of the NPs in the skeleton (represented by the femur, tibia, and fibula). The fluorescent intensities were evaluated through both NP groups (DiR-loaded PLGA NPs and DiR-loaded TC-PLGA NPs), which reached the skeleton 48 hours after intravenous administration via the tail vein in mice. After 48 hours, the 


\section{A}

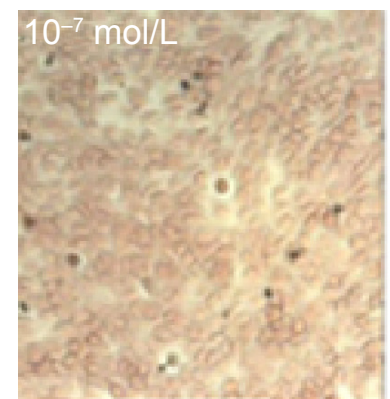

Control

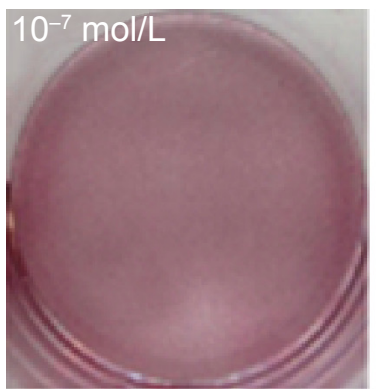

Control

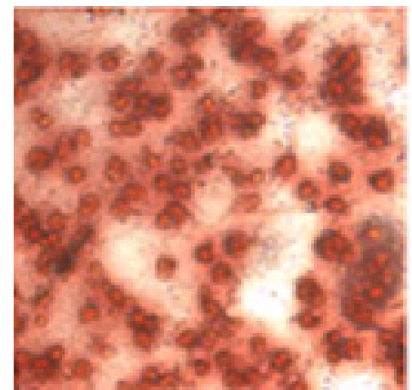

SIM/PLGA NP

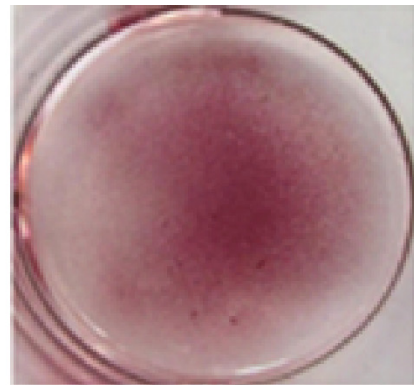

SIM/PLGA NP

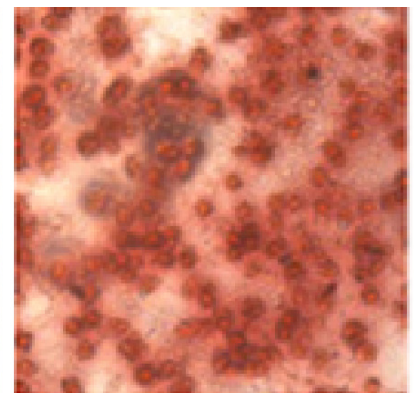

SIM/TC-PLGA NP

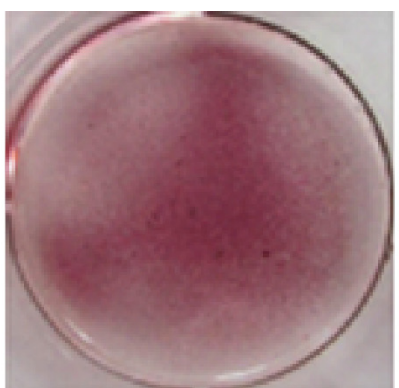

SIM/TC-PLGA NP

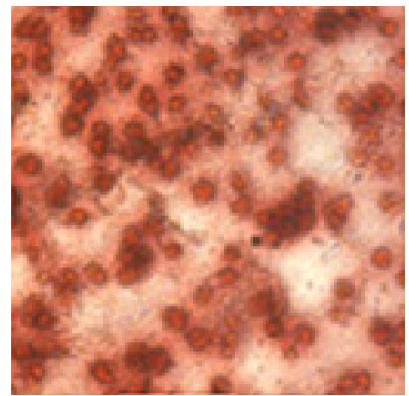

SIM

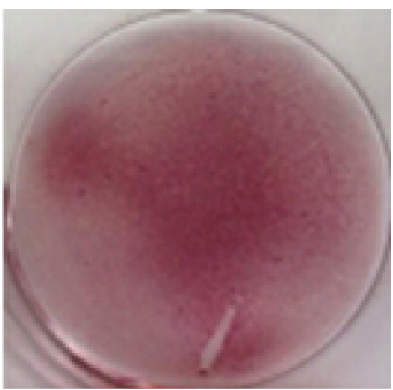

SIM

B
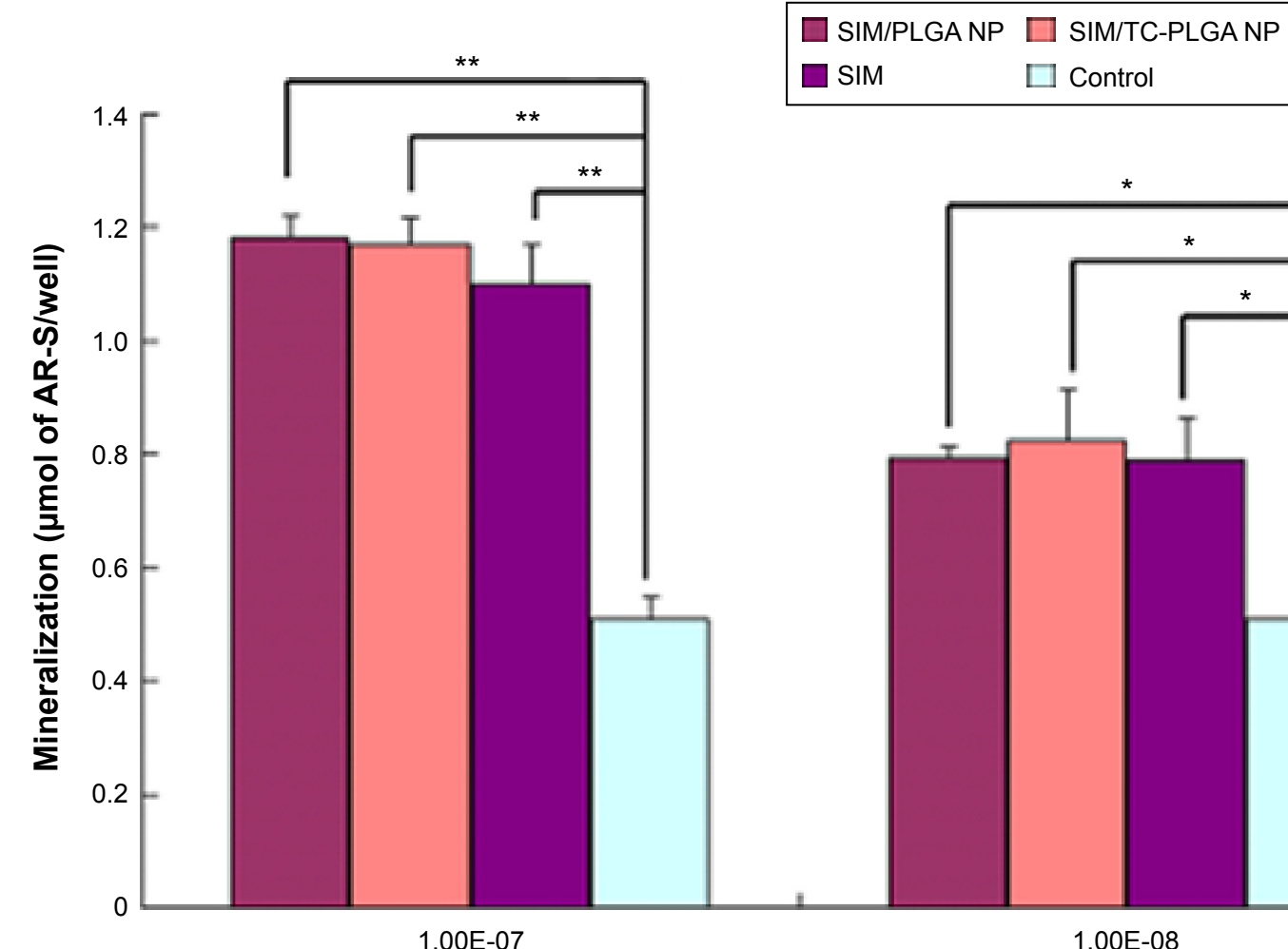

\section{Conc (mol/L)}

Figure 7 Effects of SIM/NPs on mineralization of extracellular matrix by MC3T3-EI cell culture: mineralization of extracellular matrix by optical microscope and macroscopic observation $(\mathbf{A})$, and quantitative mineralization results (B).

Notes: The data are plotted as the mean \pm SD $(n=3)$. Significant differences against controls, $* P<0.05 ; * * P<0.01$. (A) The magnification is I00 $\times$.

Abbreviations: NPs, nanoparticles; AR-S, alizarin red S dye; PLGA, poly(lactic-co-glycolic acid); SIM, simvastatin; TC-PLGA, tetracycline-poly(lactic-co-glycolic acid) moiety; Conc, concentration; SD, standard deviation. 
A

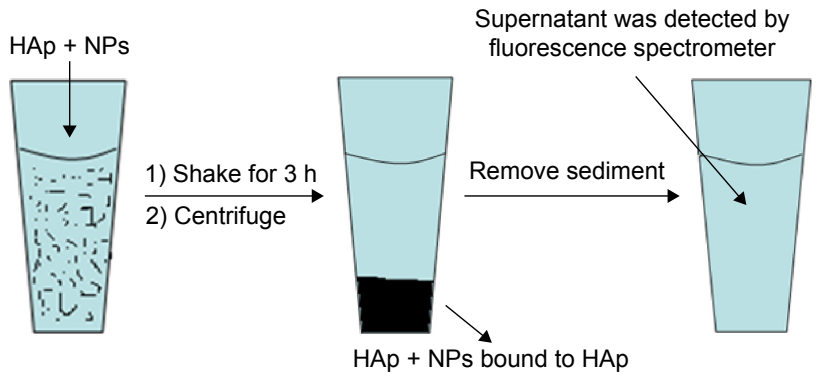

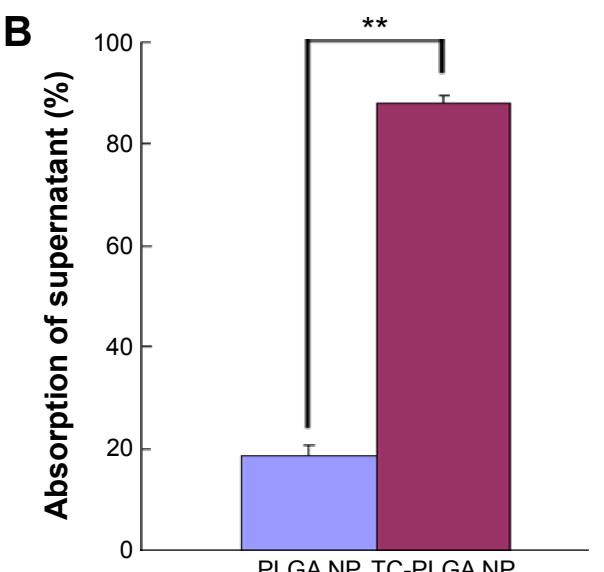

Figure 8 HAp adsorption affinity experiments using the hydrophobic fluorescent dye ODA-FITC as a drug model (A) and their results (B). Notes: The data are plotted as the mean $\pm S D(n=3)$. Significant differences against the control, $* * p<0.01$.

Abbreviations: HAp, hydroxyapatite powder; FITC, fluorescein isothiocyanate; ODA-FITC, otcadecylamine-fluorescein isothiocyanate moiety; NPs, nanoparticles; PLGA, poly(lactic-co-glycolic acid); TC-PLGA, tetracycline-poly(lactic-co-glycolic acid) moiety; SD, standard deviation; h, hours.

A

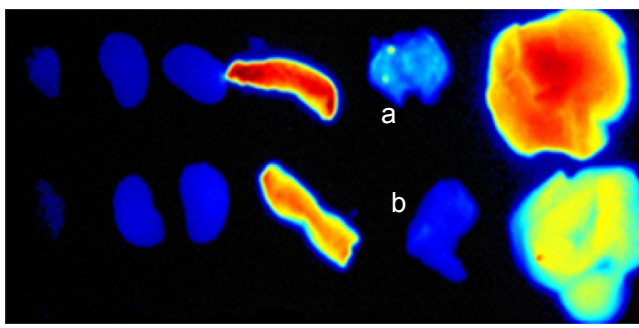

Heart Kidney Spleen

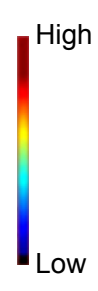

Liver
B

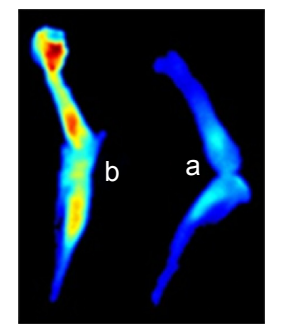

Femur, tibia, and fibula

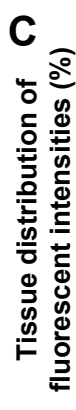

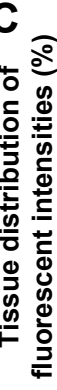

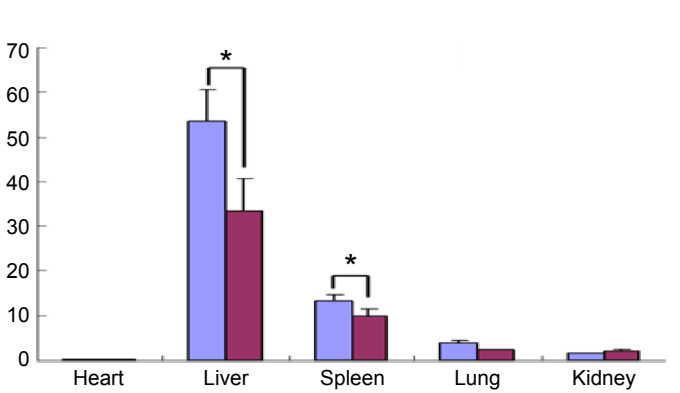

D
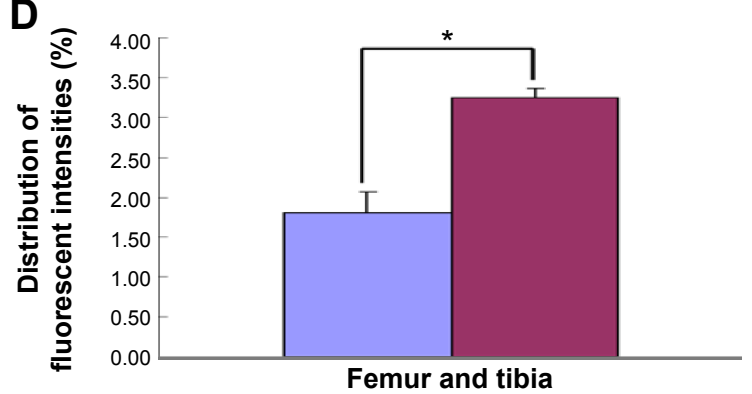

$\square$ PLGANPS $\square$ TC-PLGANPS

Figure 9 Fluorescent images of DiR-loaded PLGA NPs (a), and DiR-loaded TC-PLGA NPs taken 48 hours after nanoparticle transport into ICR mice and their quantitative results (b). Fluorescent images of the major tissues $(\mathbf{A})$, and fluorescent images of the femur, tibia, and fibula bones (B); quantitative results of the major tissues (C), and quantitative results of the femur, tibia, and fibula bones (D).

Notes: The data are plotted as the mean $\pm S D(n=3)$. Significant differences against the control, $* P<0.05$.

Abbreviations: ICR, US Institute of Cancer Research; NPs, nanoparticles; PLGA, poly(lactic-co-glycolic acid); TC-PLGA, tetracycline-poly(lactic-co-glycolic acid) moiety; SD, standard deviation. 
fluorescent intensities of DiR in the bone due to the TCPLGA NPs were clearly higher than those due to the PLGA NP control. These data were then quantified from the fluorescent intensities, and the results are shown in Figure 9D. The tissue distributions of the fluorescent intensities clearly indicated that $80.5 \%$ more DiR-loaded TC-PLGA NPs were present in the femur, tibia, and fibula than in the DiR-loaded PLGA NPs. Therefore, the TC-PLGA copolymer has a tendency to target and to accumulate in bone.

Additional in vivo tests evaluating the whole-body distribution of NPs are needed to indirectly determine the efficacy of these targeted nano-polymeric delivery systems. As shown in Figure 9A, the fluorescence signals were detected mainly in the liver and spleen, and were barely detectable in the lungs, kidneys, and heart of the mice from the PLGA NP control group. Meanwhile, the fluorescence signals observed in the spleen and liver of the mice from the TC-PLGA NP group were significantly reduced. The fluorescence signals were also barely detectable in the heart, lungs, and kidneys of the rats from the TC-PLGA NP treatment group. The reason for the accumulation of the NPs in the liver and spleen was that the macrophage cells in these organs play a role in uptake and phagocytosis. ${ }^{32}$

The whole-body distribution data were also quantified from the fluorescence intensities, and the results are shown in Figure 9C. It is worth mentioning that compared to the DiR-loaded PLGA NPs, 37.6\% and 24.0\% fewer DiR-loaded TC-PLGA NPs were present in the liver and spleen, respectively. The results showed that TC-PLGA NPs could reduce the trapping of NPs in the liver and spleen, reducing side effects in other organs caused by drugs. This can be taken as indirect evidence that TC-PLGA NPs have a tendency to target and accumulate in bone.

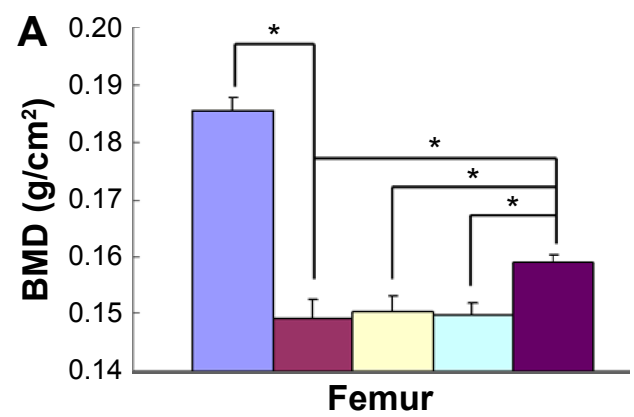

\section{Animal pharmacodynamics evaluation of bone-targeting NPs}

Twelve weeks after ovariectomy operation, four animals died, one in the SHAM group and three in ovariectomized rats. As shown in Figure 10A, the femur BMD was distinctly different between the two groups $(P<0.01)$, with values of $0.185 \pm 0.003 \mathrm{~g} / \mathrm{cm}^{2}$ in the SHAM group and $0.149 \pm 0.004 \mathrm{~g} / \mathrm{cm}^{2}$ in the OVX group, indicating the success of the osteoporotic model established in ovariectomized rats.

Eight weeks after treatment, all of the animals were sacrificed. As shown in Figure 10A, the femur BMD between groups 2 (OVX), 3 (OVX + SIM), and 4 (OVX + SIMloaded NPs) showed no significant differences $(P>0.05)$. However, compared to group 2 (OVX), the femur BMD in group 5 (OVX + SIM-loaded TC-NPs) was increased by $6.71 \%$. The recovery of BMD in the femur was $4.14 \%$ in group 3 (OVX + SIM), 2.07\% in group 4 (OVX + SIMloaded NPs), and $17.59 \%$ in group 5 (OVX + SIM-loaded TC-NPs).

The caput femoris BMD was also measured, as shown in Figure 10B. A statistically significant increase in the caput femoris BMD was observed. The caput femoris BMD was increased by $9.47 \%$ in group 3 (OVX + SIM), $7.92 \%$ in group 4 (OVX + SIM-loaded NPs), and $17.04 \%$ in group 5 (OVX + SIM-loaded TC-NPs) when compared to the group 2 (OVX) group. The recovery of the caput femoris BMD was $35.26 \%$ in group 3 (OVX + SIM), 29.49\% in group 4 (OVX + SIM-loaded NPs), and $63.46 \%$ in group 5 (OVX + SIM-loaded TC-NPs). The better recovery of the caput femoris from osteoporosis may be because cancellous bone structures such as the caput femoris are more sensitive than cortical bone to the inducement of osteoblast differentiation.

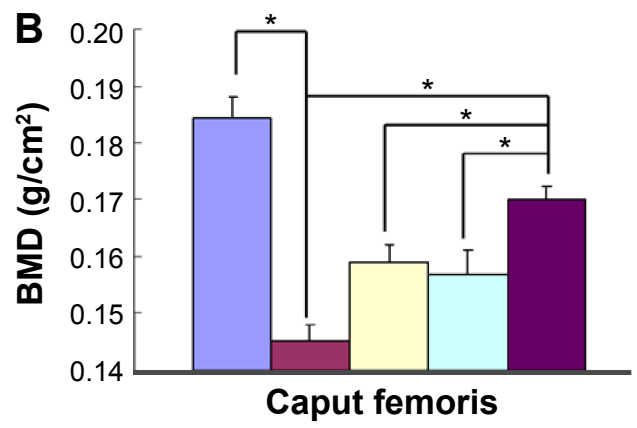

$\square$ SHAM $\square$ OVX $\square$ SIM $\sqsubset$ SIM/PLGA NPs $\square$ SIM/TC-PLGA NPS

Figure 10 Bone mineral density (BMD) of the femur (A) and caput femoris (B) in ovariectomized Sprague Dawley rats after 8 weeks of treatment.

Notes: The data are plotted as the mean \pm SD $(n=4)$. Significant differences against the control, $* P<0.05$.

Abbreviations: NPs, nanoparticles; PLGA, poly(lactic-co-glycolic acid); SIM, simvastatin; TC-PLGA, tetracycline-poly(lactic-co-glycolic acid) moiety; SHAM, control shamoperated rats; OVX, ovariectomized rats; SD, standard deviation. 
Overall, the results demonstrated that the TC-PLGA NPs have a good ability to target bone and to achieve high bone tissue drug accumulation, improving the curative effects of SIM on osteoporosis. These NPs may act as a delivery system for hydrophobic drugs and are expected to reduce the required drug dose administered or to reduce the side effects of drugs in other organs, thereby improving the curative effects of SIM.

\section{Conclusion}

Two types of NPs (PLGA NPs and TC-PLGA NPs) were designed and prepared, and then their bone-targeting ability and curative effects on osteoporosis were assessed. ${ }^{1} \mathrm{H}-\mathrm{NMR}$ was used to confirm that the final TC-PLGA ${ }_{2000}$ product was successfully synthesized. The average particle size was approximately $220 \mathrm{~nm}$ for the two types of copolymer NPs, and the SIM-loaded NPs exhibited a cumulative release of up to $80 \%$ within 72 hours in vitro. The in vitro cell evaluation indicated that the NPs had excellent cellular uptake ability and great biocompatibility with MC3T3-E1 cells, which would reduce the cytotoxic effects of SIM. The cell mineralization assay showed that the SIM-loaded NPs induced osteogenic differentiation and mineralized nodule formation in MC3T3-E1 cells, thus achieving the same effect as SIM. In addition, the TC moiety of the TC-PLGA NPs had enhanced bone-binding capacity when binding to apatite mineral surfaces, due to its affinity for HAp.

Biophotonic imaging technology was also used to examine the organ distribution of the DiR-labeled areas or of the fluorescent dye delivered by the PLGA and TC-PLGA NPs in ICR mice. The intensity of the intraosseous fluorescence signal was stronger in the bone after injection with the TC-PLGA NPs than after treatment with the PLGA NPs. The animal pharmacodynamics of treatment of osteoporosis in ovariectomized rats suggested that the SIM-loaded TC-PLGA NPs could improve the curative effects of SIM on the recovery of DMD compared to either the SIM-loaded PLGA NPs or free SIM. The bone-targeting NPs, based on the conjugation of TC to PLGA copolymers, had the ability to target bone, and their use for the treatment of osteoporosis with SIM was successfully achieved. These NPs may act as a delivery system for hydrophobic drugs and are expected to reduce the drug dose required for administration or to reduce the side effects in other organs, thereby improving the curative effects of SIM.

\section{Disclosure}

The authors report no conflicts of interest in this work.

\section{References}

1. Mundy G, Garrett R, Harris S, et al. Stimulation of bone formation in vitro and in rodents by statins. Science. 1999;286(5446):1946-1949.

2. Blum CB. Comparison of properties of four inhibitors of 3-hdroxy-3methyglutary-coenzyme A reductase. Am J Cardiol. 1994;73:3D-11D.

3. Montagnani A, Gonnelli S, Cepollaro C, et al. Effect of simvastatin treatment on bone mineral density and bone turnover in hypercholesterolemic postmenopausal women: a 1-year longitudinal study. Bone. 2003;32:427-433.

4. Tikiz C, Tikiz H, Taneli F, Gumuser G, Tuzun C. Effects of simvastatin on bone mineral density and remodeling parameters in postmenopausal osteopenic subjects: 1-year follow-up study. Clin Rheumatol. 2005;24: 447-452.

5. Skoglund B, Forslund C, Aspenberg P. Simvastatin improves fracture healing in mice. J Bone Miner Res. 2002;17:2004-2008.

6. Guyton JR. Benefit versus risk in statin treatment. Am J Cardiol. 2006;97: 95C-97C.

7. Shekunov BY, Chattopadhyay P, Seitzinger J, Huff R. Nanoparticles of poorly water-soluble drugs prepared by supercritical fluid extraction of emulsions. Pharm Res. 2006;23(1):196-204.

8. Kwon GS, Kataoka K. Block copolymer micelles as long-circulating drug vehicles. Adv Drug Deliv Rev. 1995;16(2):295-309.

9. Kataoka K, Harada A, Nagasaki Y. Block copolymer micelles for drug delivery: design, characterization and biological significance. Adv Drug Deliv Rev. 2001;47(1):113-131.

10. Hans ML, Lowman AM. Biodegradable nanoparticles for drug delivery and targeting. Curr Opin Solid State Mater Sci. 2002;6(4):319-327.

11. De Jong WH, Borm PJ. Drug delivery and nanoparticles: applications and hazards. Int J Nanomedicine. 2008;3(2):133.

12. Wang G, Uludag H. Recent developments in nanoparticle-based drug delivery and targeting systems with emphasis on protein-based nanoparticles. Expert Opin Drug Deliv. 2008;5:499-515.

13. Danhier F, Ansorena E, Silva JM, Coco R, Le Breton A, Préat V. PLGA-based nanoparticles: an overview of biomedical applications. $J$ Control Release. 2012;161(2):505-522.

14. Ho ML, Fu YC, Wang GJ, et al. Controlled release carrier of BSA made by W/O/W emulsion method containing PLGA and hydroxyapatite. $J$ Control Release. 2008;128(2):142-148.

15. Perrin RA. Binding of tetracycline to bone. Nature. 1965;208(11): 787-788.

16. Mazaleyrat JP, Boutboul A, Lebars Y, Gaucher A, Wakselman M. Practical resolution of an atropoisomeric [alpha],[alpha]-disubstituted glycine with 1-phenylalanine cyclohexylamide as chiral auxiliary. Tetrahedron Asymmetry. 1998;9(15):2701-2713.

17. Gaucher A, Zuliani Y, Cabaret D, Wakselman M, Mazaleyrat JP. Synthesis and resolution of $\beta$ 2, 2-HBin, the first enantiomerically stable $\beta$-amino acid with chirality only due to axial dissymmetry. Tetrahedron Asymmetry. 2001;12(18):2571-2580.

18. Nath SD, Son S, Sadiasa A, Min YK, Lee BT. Preparation and characterization of PLGA microspheres by the electrospraying method for delivering simvastatin for bone regeneration. Int $J$ Pharm. 2013;443(1-2):87-94.

19. Ho MH, Chiang CP, Liu YF, et al. Highly efficient release of lovastatin from poly(lactic-co-glycolic acid) nanoparticles enhances bone repair in rats. J Orthop Res. 2011;29(10):1504-1510.

20. Tiwari R, Pathak K. Nanostructured lipid carrier versus solid lipid nanoparticles of simvastatin: comparative analysis of characteristics, pharmacokinetics and tissue uptake. Int J Pharm. 2011;415(1): 232-243.

21. Wang CZ, Fu YC, Jian SC, et al. Synthesis and characterization of cationic polymeric nanoparticles as simvastatin carriers for enhancing the osteogenesis of bone marrow mesenchymal stem cells. $J$ Colloid Interface Sci. 2014;432:190-199.

22. Yang F, Zhao S, Zhang F, He FM, Yang GL. Simvastatin-loaded porous implant surfaces stimulate preosteoblasts differentiation: an in vitro study. Oral Surg Oral Med Oral Pathol Oral Radiol Endod. 2011; 111(5):551-556. 
23. Park JB, Zhang H, Lin CY, et al. Simvastatin maintains osteoblastic viability while promoting differentiation by partially regulating the expressions of estrogen receptors $\alpha . J$ Surg Res. 2012;174(2): 278-283.

24. Gregory CA, Gunn WG, Peister A, Prockop DJ. An Alizarin red-based assay of mineralization by adherent cells in culture: comparison with cetylpyridinium chloride extraction. Anal Biochem. 2004;329(1): 77-84.

25. Maeda T, Matsunuma A, Kawane T, Horiuchi N. Simvastatin promotes osteoblast differentiation and mineralization in MC3T3-E1 cells. Biochem Biophys Res Commun. 2001;280(3):874-877.

26. Ho ML, Fu YC, Wang GJ, et al. Controlled release carrier of BSA made by $\mathrm{W} / \mathrm{O} / \mathrm{W}$ emulsion method containing PLGA and hydroxyapatite. J Control Release. 2008;128:142-148.

27. Fu YC, Fu TF, Wang HJ, et al. Aspartic acid-based modified PLGAPEG nanoparticles for bone targeting: In vitro and in vivo evaluation. Acta Biomater. 2014;10(11):4583-4596.
28. Waynforth HB. Experimental and Surgical Technique in the Rat. Academic Press, London: Academic Press; 1980.

29. Wang JW, Xu SW, Yang DS, Lv RK. Locally applied simvastatin promotes fracture healing in ovariectomized rat. Osteoporos Int. 2007;18(12): 1641-1650.

30. Ito T, Takemasa M, Makino K, Otsuka M. Preparation of calcium phosphate nanocapsules including simvastatin/deoxycholic acid assembly, and their therapeutic effect in osteoporosis model mice. $J$ Pharm Pharmacol. 2013;65(4):494-502.

31. Maeda T, Matsunuma A, Kurahashi I, Yanagawa T, Yoshida H, Horiuchi N. Induction of osteoblast differentiation indices by statins in MC3T3-E1 cells. J Cell Biochem. 2004;92(3):458-471.

32. Wong L, Bendayan R, Rauth AM, Li YQ, Wu XY. Chemotherapy with anticancer drugs encapsulated in solid lipid nanoparticles. Adv Drug Deliv Rev. 2007;59(6):491-504.
International Journal of Nanomedicine

\section{Publish your work in this journal}

The International Journal of Nanomedicine is an international, peerreviewed journal focusing on the application of nanotechnology in diagnostics, therapeutics, and drug delivery systems throughout the biomedical field. This journal is indexed on PubMed Central, MedLine, CAS, SciSearch $®$, Current Contents $\AA /$ Clinical Medicine,

\section{Dovepress}

Journal Citation Reports/Science Edition, EMBase, Scopus and the Elsevier Bibliographic databases. The manuscript management system is completely online and includes a very quick and fair peer-review system, which is all easy to use. Visit http://www.dovepress.com/ testimonials.php to read real quotes from published authors.

Submit your manuscript here: http://www.dovepress.com/international-journal-of-nanomedicine-journal 